\title{
Metrological Properties of a Two-Output Transducer for Measuring Sum and Difference of Small Resistances
}

\author{
Adam Idzkowski ${ }^{1}$, Wojciech Walendziuk ${ }^{1}$, Pawel Swietochowski ${ }^{2}$, Zygmunt Warsza ${ }^{3}$ \\ ${ }^{1}$ Department of Electrical Engineering, Bialystok University of Technology, \\ Wiejska 45D St., 15-351 Bialystok, Poland \\ ${ }^{2}$ Mektron, Bialystok, Poland \\ ${ }^{3}$ Industrial Research Institute for Automation and Measurements PIAP, \\ Warsaw, Poland \\ a.idzkowski@pb.edu.pl
}

\begin{abstract}
It presents an alternative realization of a circuit which supplies a resistance bridge, which has the form of a quadrilateral, equipped with two current sources and four electronic keys. The idea with alternately switched current sources allows to measure simultaneously small (up to $1 \Omega$ ) differences and sums of two changes in resistance taken from a pair of sensors plugged in the circuit. Problems relating to circuit realization and a way of signal processing are discussed. In the prototype transducer shielding and filtering RFI/EMI were used. The results of measurements conducted with the use of precision resistance decade boxes are presented.
\end{abstract}

Index Terms-Resistive transducers; sensors systems; measurement techniques; signal sampling; noise cancellation.

\section{INTRODUCTION}

Resistive sensors are the most commonly used passive sensors whose resistance changes according to some environmental effect. A pair of resistive sensors produces two changes in resistance which can be converted into voltage or current and finally - into a digital form [1].

In a one-parameter measurement a resistive sensor is a part of a resistance bridge. It aims at forming an analogue output voltage which is properly conditioned. The unbalanced resistive bridges are discussed in detail in [2], [3]. These bridge circuits are preferred in dynamic measurements where the frequency band is wide and, even with a big change in the sensor resistance, the amplifier output voltage is not in saturation.

It happens frequently in practice that there is a need of two-quantity measurement. Both quantities have a simultaneous influence on sensors but their resistances change in a different way. It is realized by circuits where two parameters (resistances) are measured on terminals, and their ratio [4] or difference [5] is determined. There are applications where a sum and a difference of changes in resistance are obtained by measuring voltages at several

Manuscript received 7 March, 2017; accepted 14 August, 2017.

The paper was prepared at Bialystok University of Technology within a framework of the S/WE/1/2013 project funded by Ministry of Science and Higher Education. independent outputs of bridge-circuits [6].

Some tests on the original transducer, which consists of a resistance bridge in the form of a quadrilateral supplied by one current source in an unconventional way, will be introduced in this paper. This solution allows to measure a sum and a difference of two changes in resistance. It is a deployment and completion of research which was described earlier in paper [7] and it was mainly dedicated to amplifying stages of the transducer. A general idea of a switched bridge-circuit was presented in [8]. In another paper [9] a simple model of this transducer and its application with a pair of sensors acting as strain gauges and RTD's was presented. The conducted experiment confirmed a possibility of measuring strain and temperature simultaneously with limited error $\pm 1.8^{\circ} \mathrm{C}$. This model, and especially its signal conditioning part, which consisted of low-pass filters, differential amplifiers and voltage dividers (to reduce high bipolar offsets of output voltages), was not optimal. Accordingly, in this paper another model and a signal conditioning technique is presented. It allows to eliminate these segments in output signals (peaks) which are caused by commutation of the MOSFET transistors. This causes higher output voltage sensitivity and a better resolution of measured changes in resistance can be gained.

\section{TwO-OUTPUT R/U TRANSDUCER}

The idea of this solution lies in the use of one current source LT3092DC rapidly switched by means of electronic keys ( 2 pcs. Si7143DP and 2 pcs. SiR484DP MOSFET transistors). The constant current $I_{\mathrm{s}}$ is alternately brought to the nodes A and B (Fig. 1).

The potential differences $U_{A B}$ and $U_{D C}$ are measured and averaged on the diagonals of the bridge-circuit.

The voltage samples $U_{A B_{-} \text {amp } i}$ and $U_{D C_{-} \text {amp } i}$ after amplification by measuring amplifiers AD8221AR in two consecutive sequences of operation Q1, Q3 ON (PI) and Q2, Q4 ON (PII) are averaged (Fig. 1):

$$
U_{D C_{-} \text {amp } i}=\frac{U_{D C_{-} a m p(P I) i}+U_{D C_{-} a m p(P I I) i}}{2},
$$




$$
U_{A B_{-} a m p i}=\frac{U_{A B_{-} a m p(P I) i}+U_{A B_{-} a m p(P I I) i}}{2} .
$$

Conduction resistance of the electronic keys (low $\left.R_{D S(\mathrm{on})}\right)$ is small and different for each element.
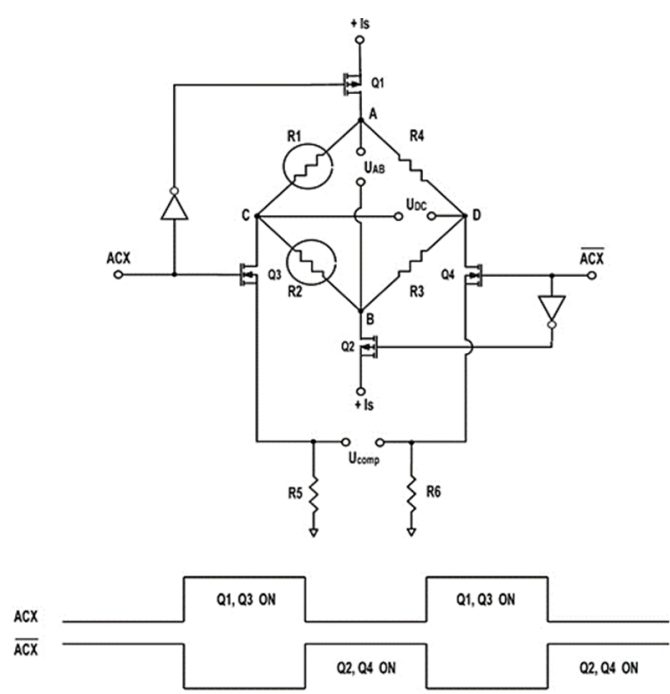

Fig. 1. Bridge-circuit with two analogue outputs supplied by two switched current sources [7].

Therefore, an asymmetry due to different voltage drops on the keys is possible, however it has no significance for the operation of the circuit supplied from a current source.

Two resistors $R_{1}$ and $R_{2}$ represent sensors with equal nominal resistance $R_{10}$. Their absolute changes in resistance are $\Delta R_{1}$ and $\Delta R_{2}$. Assuming that other resistances of the bridge-circuit are not changed, i.e.: $R_{3}=R_{0}, R_{4}=R_{0}$ (their changes are $\left.\Delta R_{3}=\Delta R_{4}=0\right)$. If modules of the values $\left|\Delta R_{1}\right|$, $\left|\Delta R_{2}\right|$ are small enough $(\leq 1 \Omega)[7]$ and all initial resistances are equal $R_{1}=R_{2}=R_{3}=R_{4} \equiv R_{0}$, (1) and (2) can be written as linear functions of sum $R_{D C}=\Delta R_{1}+\Delta R_{2}$ and difference $R_{A B}=\Delta R_{1}-\Delta R_{2}$ of changes in resistance:

$$
\begin{gathered}
U_{D C_{-} \text {amp }} \cong 0.125 \times I_{s} \times G \times\left(\Delta R_{1}+\Delta R_{2}\right)= \\
=0.125 \times I_{s} \times G \times R_{D C}, \\
U_{A B_{-} a m p} \cong 0.125 \times I_{S} \times G \times\left(\Delta R_{1}-\Delta R_{2}\right)= \\
=0.125 \times I_{S} \times G \times R_{A B},
\end{gathered}
$$

where $I_{S}$ - supplying current, $G$ - gain of amplifiers.

The main converter components are marked in Fig. 2.

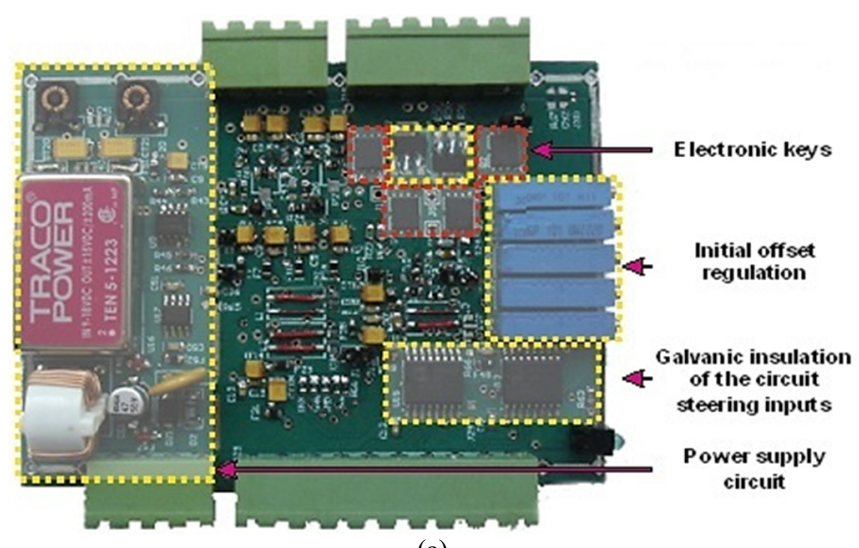

(a)

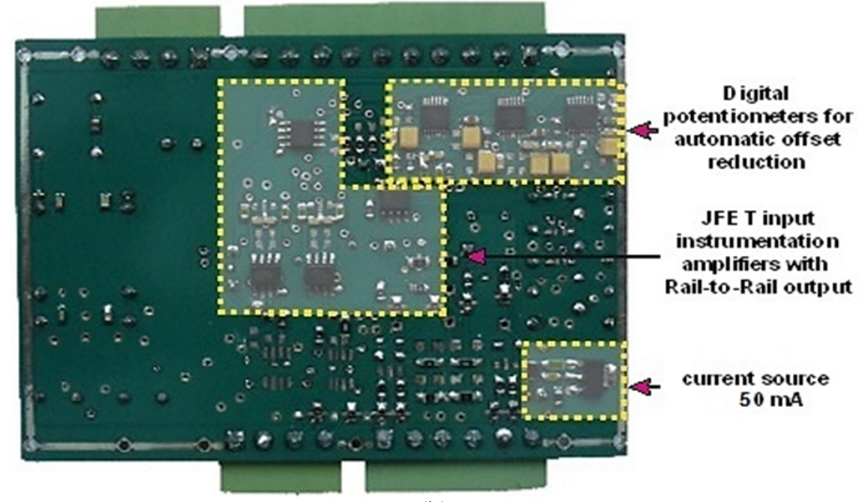

(b)

Fig. 2. The real model of $\mathrm{R} / \mathrm{U}$ transducer with power supply and connection terminals (left side - power supply, right side - measuring circuit).

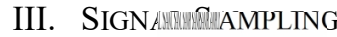

The electronic keys in thrmmin/U transducer (Fig. 3) are controlled by means of a messurwement card (NI USB-6341) and digital signals ACX and $\bar{m} \bar{x}$ i range $1 \mathrm{~Hz} \ldots 250 \mathrm{~Hz}$, dewwert in the parameters of the DAQ program.

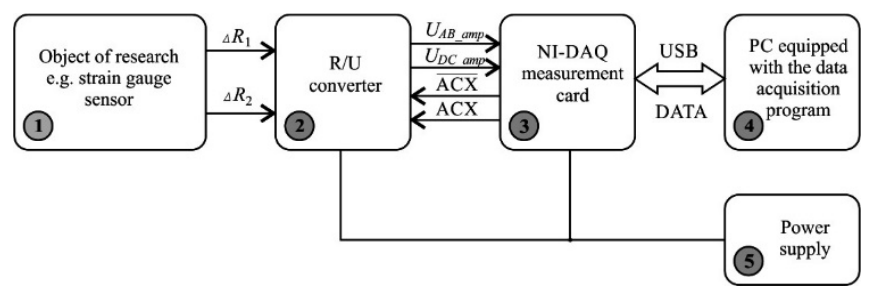

Fig. 3. Measuring system with $\mathrm{R} / \mathrm{U}$ transducer, measuring card and computer with a data acquisition program.

The synchronization signal SYNC is simultaneously generated with the ACX signal. Both signals have identical polarity (Fig. 4). The SYNC signal frequency is twice higher $f_{S Y N C}=2 f_{A C X}$. The purpose of this signal is to synchronize ADC (analog-to-digital converter) in the measurement card with the system which switches the control keys.

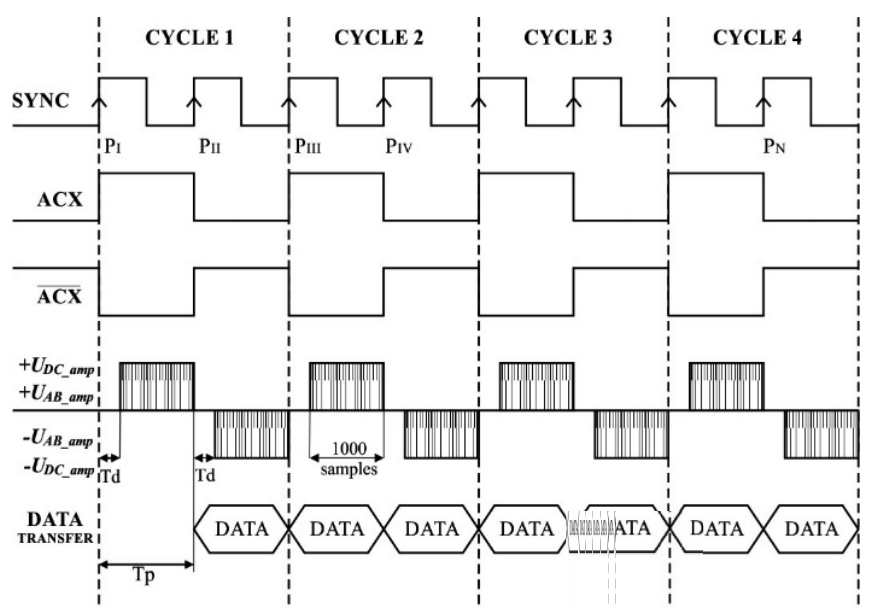

Fig. 4. Timing diagram, SYNC signal, ACX and $\bar{m} \bar{n} \overline{x i} \bar{x}$.

The ADC works at a finite operation mode, which means that it samples a defined number of samples $(N=1000)$ and it is automatically turned off. The ADC is triggered by a rising edge of SYNC signal but after a release its work is delayed for the time $T_{d}(1 \mathrm{~ms}$ fixed). 
The rising edge of SYNC signal is also the moment when the electronic keys are switched. For this reason, a delay of sampling by the ADC is needed to suppress the transient state periods of time in the measured signals caused by commutation (Fig. 5).

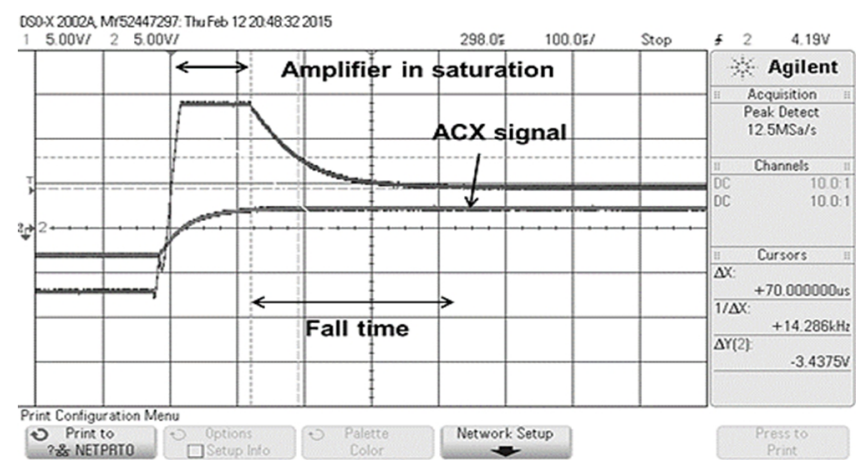

Fig. 5. Settling time of the voltage $U_{A B}$ after electronic keys are switched on, the amplifiers are saturated $120 \mu \mathrm{s}, 0 \%-100 \%$ fall time: $300 \mu \mathrm{s}$.

The ADC sampling rate is dependent on the switching frequency $f_{A C X}$

$$
f_{A D C}=\frac{N}{\frac{1}{2 f_{A C X}}-T_{d}} .
$$

The ACD adjusts $f_{A D C}$ to get 1000 samples for every sequence during switching.

The measured voltages (1) and (2) are the result of arithmetic operations on four vectors of data. There are $N=1000$ samples in each measurement sequence. For the first (PI) and the second (PII) sequences the mean values are obtained in the following way:

$$
\begin{aligned}
U_{D C_{-} \text {amp }} & =\sum_{i=1}^{N} \frac{U_{D C_{\_} a m p(\mathrm{PI}) i}+U_{D C_{-} \_a m p(\mathrm{PII}) i}}{2 N}, \\
U_{A B_{-} \_a m p} & =\sum_{i=1}^{N} \frac{U_{A B_{-} a m p(\mathrm{PI}) i}+U_{A B_{\_} a m p(\mathrm{PII}) i}}{2 N} .
\end{aligned}
$$

The subsequent values are calculated at the next switching sequences, i.e. time series $U_{D C_{-} \text {amp(PIII) }}$ and $U_{A B \_a m p(P I I I)}$ are created, and the averaging is done for the second (PII) and third (PIII) switching sequences. Then for the third (PIII) and fourth (PIV) ones, etc. The results $\left(R_{A B}\right.$ and $R_{D C}$ ) are displayed with a delay of $T_{p}=1 / 2 f_{A C X}$ (Fig. 4 ).

\section{Filtering, SHIELding THE CiRCUIT AND THERMAL DRIFT}

Two measuring channels of the tested $\mathrm{R} / \mathrm{U}$ transducer are composed of a bridge-circuit (Fig. 1), a preamplifier and an instrumental amplifier. They are presented in publication [7]. The system was tested by setting the gain $G=250$ by using precision resistors $\left(100 \Omega, 0.1 \%, \mathrm{TC}=25 \mathrm{ppm} /{ }^{\circ} \mathrm{C}\right)$ at the instrumental amplifiers in measurement channels $\mathrm{AB}$ and DC. The single-pole low-pass RC filters $f_{3 d B}=4 \mathrm{kHz}$ were applied just behind the bridge-circuit at the entrance to the preamplifier. The tests demonstrated that the use of filter in this place is essential for this transducer. The outcome was 5-time lower noise (from $479 \mathrm{mV}$ to $94 \mathrm{mV}$ peak-to- peak, Fig. 6).

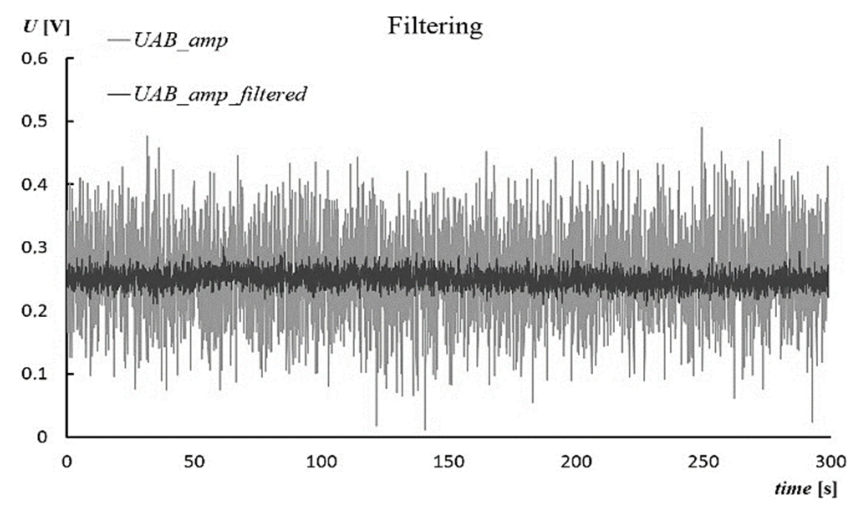

Fig. 6. Filtering effect (5 times lower noise).

The module from Fig. 2 was encased with the use of a copper shield and painted in matt black. The connections between the measuring card and the ADC and between the $\mathrm{ADC}$ and the decade box resistors were made with the use of an LIYCY $4 \times 0.34 \mathrm{~mm}^{2}$ shielded cable. The signal from the measurement channel $\mathrm{AB}$ after filtering and shielding is shown in Fig. 7.

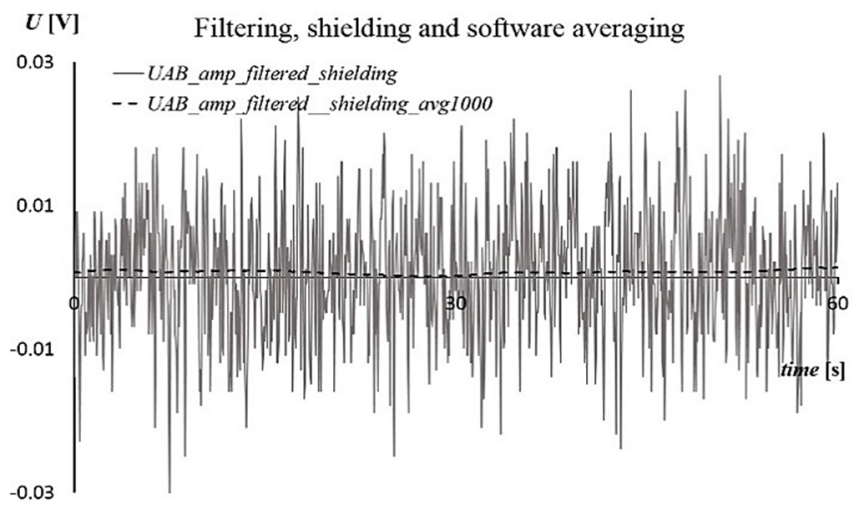

Fig. 7. Filtering, shielding and software averaging effect.

Figure 7 shows the $56 \mathrm{mV}$ peak-to-peak noise. The value is similar in channel DC. Finally, when software averaging was applied (1000 samples of data are averaged) [10], the noise was reduced to $2 \mathrm{mV}$ peak-to peak.

These results were obtained after self-heating of the device. As shown in Fig. 8, the temperature of this module increases by approx. $12{ }^{\circ} \mathrm{C}$ for about 15 minutes-20 minutes.

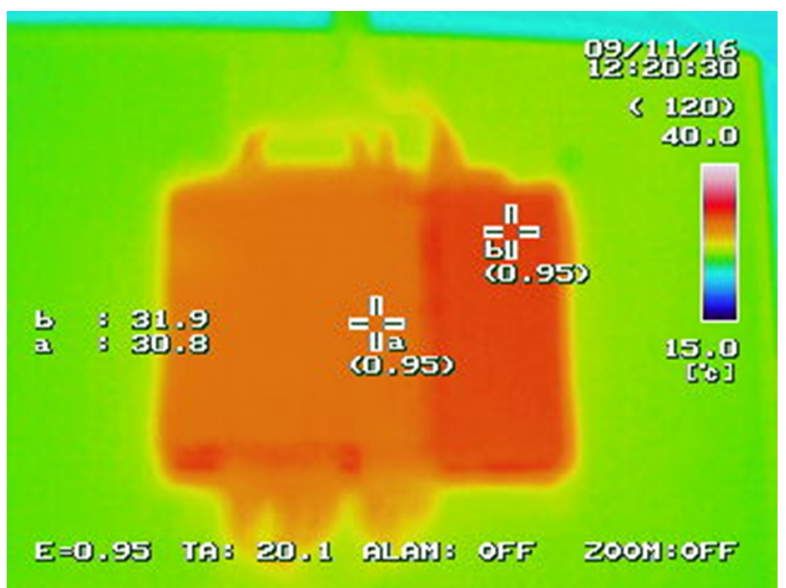

Fig. 8. Thermal image of working device - temperatures are $10.7^{\circ} \mathrm{C}$, $11.8^{\circ} \mathrm{C}$ higher than ambient temperature. 
At this time, the temperature distribution of a PCB is uniform. The design of the system (supply and measuring system on one common plate) causes thermal drifts in the outputs (Fig. 9).

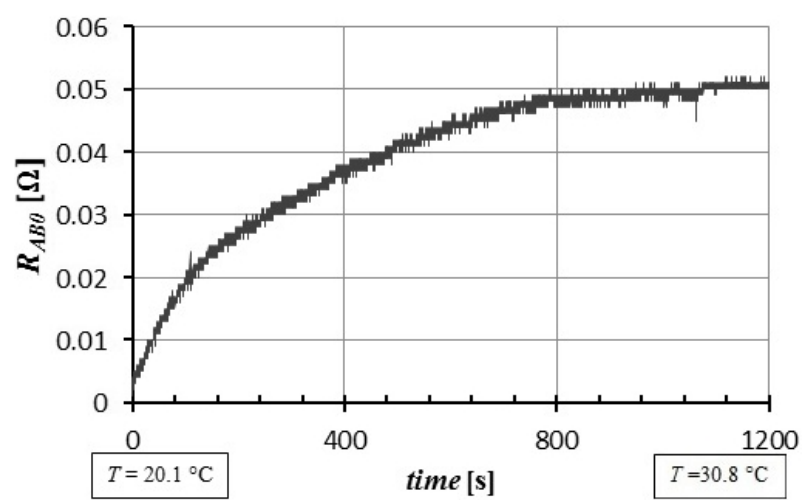

When self-heating is completed the values in outputs are stable and their offsets are nulled (in the software).

\section{Metrological Properties}

The measuring range of the transmitter is $\pm 1 \Omega$. With the previously described filtering, shielding and software averaging, it was possible to read the results with the resolution of $0.001 \Omega$.

The transducer was tested with the use of two decade resistors MDR-93/2-4aa. They had resolution of $0.01 \Omega$, the accuracy class was less than $0.05 \%$ and the thermal coefficient $\mathrm{TC}<0.002 \% /{ }^{\circ} \mathrm{C}$ (Fig. 10).

As shown in Fig. 11, the successive changes in resistance $\left|R_{A B d}\right|=0.01 \Omega$, which were set by a resistance decade box, caused the voltage increments. The settling time of the voltage was about $50 \mathrm{~ms}$.

Fig. 9. Thermal drift of $R_{A B O}$ (the initial value of $R_{A B}$ ) caused by internal heating of device.

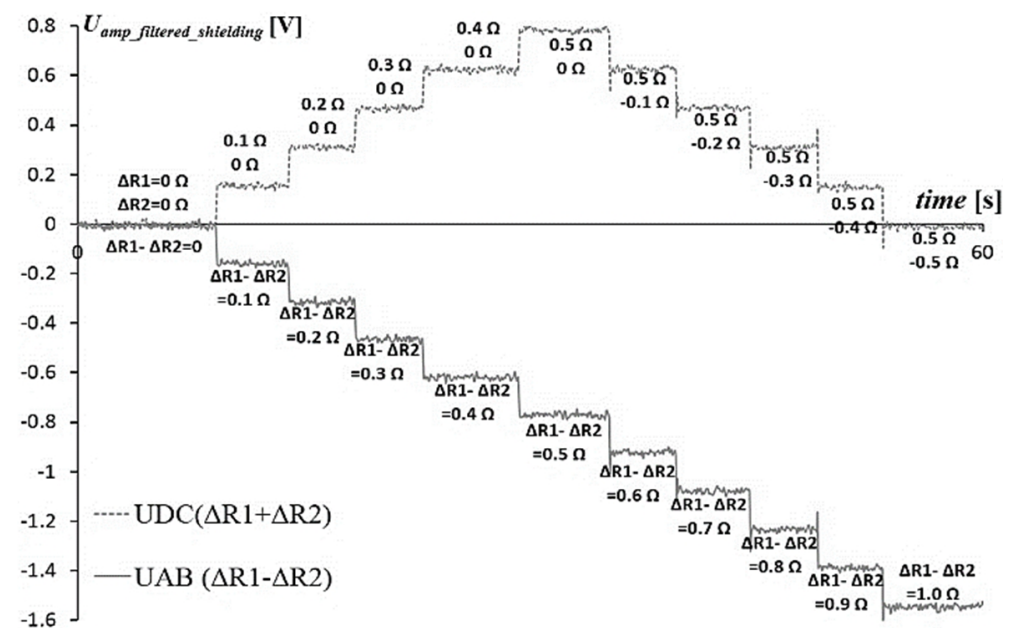

Fig. 10. Test with two MDR-93/2-4aa resistance decade boxes (for whole measurement range, the voltages filtered and not averaged in software).

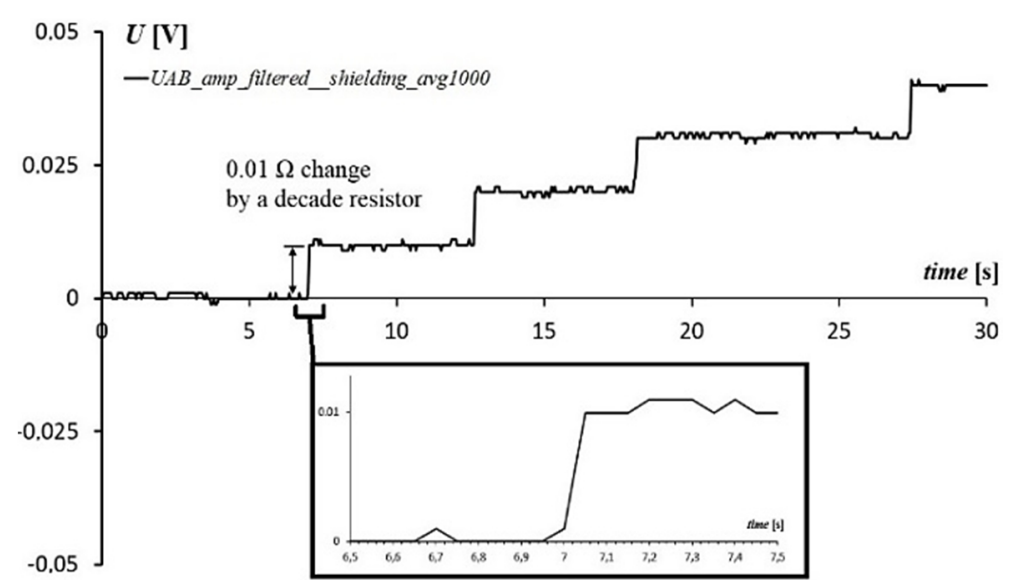

Fig. 11. Settling time of $U_{A B}(0.01 \Omega$ changes set by a decade resistor).

\section{RESULTS}

The sums $R_{D C m}$ and differences $R_{A B m}$ of changes in resistance were registered when $f_{A C X}=10 \mathrm{~Hz}$. The appropriate sums $R_{D C d}$ and differences $R_{A B d}$ were set by a pair of resistance decade boxes MDR-93/2-4aa. The modules of absolute differences $\left|R_{A B}\right|=\left|R_{A B m}-R_{A B d}\right|$ and $\left|R_{D C}\right|$ $=\left|R_{D C m}-R_{D C d}\right|$ are presented in Table I-Table IV for different $R_{1}$ and $R_{2}$ settings. The biggest difference was $0.085 \Omega$ (Table III).
TABLE I. RESULTS WHEN $R_{1} \geq R_{2}$ AND $R_{2}=$ CONST.

\begin{tabular}{|c|c|c|c|c|c|c|c|}
\hline $\boldsymbol{R}_{\mathbf{1}}$ & $\boldsymbol{R}_{\mathbf{2}}$ & $\boldsymbol{R}_{\boldsymbol{A B m}}$ & $\boldsymbol{R}_{\boldsymbol{A B d}}$ & $\boldsymbol{R}_{\boldsymbol{D} \boldsymbol{m} \boldsymbol{m}}$ & $\boldsymbol{R}_{\boldsymbol{D} C \boldsymbol{d}}$ & $\left|\Delta \boldsymbol{R}_{\boldsymbol{A B}}\right|$ & $\left|\Delta \boldsymbol{R}_{D C}\right|$ \\
\hline$\Omega$ & $\Omega$ & $\Omega$ & $\Omega$ & $\Omega$ & $\Omega$ & $\Omega$ & $\Omega$ \\
\hline 100.00 & 100.00 & 0.000 & 0.000 & 0.000 & 0.000 & 0.000 & 0.000 \\
\hline 100.20 & 100.00 & 0.198 & 0.200 & 0.190 & 0.200 & 0.002 & 0.010 \\
\hline 100.40 & 100.00 & 0.399 & 0.400 & 0.381 & 0.400 & 0.001 & 0.019 \\
\hline 100.60 & 100.00 & 0.600 & 0.600 & 0.575 & 0.600 & 0.000 & 0.025 \\
\hline 100.80 & 100.00 & 0.803 & 0.800 & 0.770 & 0.800 & 0.003 & 0.030 \\
\hline 101.00 & 100.00 & 1.007 & 1.000 & 0.964 & 1.000 & 0.007 & 0.036 \\
\hline
\end{tabular}


TABLE II. RESULTS WHEN $R_{1} \geq R_{2}$ AND $R_{1}=$ CONST.

\begin{tabular}{|c|c|c|c|c|c|c|c|}
\hline $\boldsymbol{R}_{\mathbf{1}}$ & $\boldsymbol{R}_{\mathbf{2}}$ & $\boldsymbol{R}_{\boldsymbol{A B m}}$ & $\boldsymbol{R}_{\boldsymbol{A B d}}$ & $\boldsymbol{R}_{\boldsymbol{D C} \boldsymbol{m}}$ & $\boldsymbol{R}_{\boldsymbol{D C d}}$ & $\mid \boldsymbol{\Delta \boldsymbol { R } _ { \boldsymbol { A B } } |}$ & $\mid \boldsymbol{\Delta \boldsymbol { R } _ { \boldsymbol { D C } } |}$ \\
\hline $\boldsymbol{\Omega}$ & $\boldsymbol{\Omega}$ & $\Omega$ & $\Omega$ & $\Omega$ & $\Omega$ & $\Omega$ & $\Omega$ \\
\hline 100.00 & 100.00 & -0.001 & 0.000 & -0.001 & 0.000 & 0.001 & 0.001 \\
\hline 100.00 & 99.80 & 0.192 & 0.200 & -0.215 & -0.200 & 0.008 & 0.015 \\
\hline 100.00 & 99.60 & 0.386 & 0.400 & -0.429 & -0.400 & 0.014 & 0.029 \\
\hline 100.00 & 99.40 & 0.577 & 0.600 & -0.645 & -0.600 & 0.023 & 0.045 \\
\hline 100.00 & 99.20 & 0.769 & 0.800 & -0.861 & -0.800 & 0.031 & 0.061 \\
\hline 100.00 & 99.00 & 0.958 & 1.000 & -1.081 & -1.000 & 0.042 & 0.081 \\
\hline
\end{tabular}

TABLE III. RESULTS WHEN $R_{1} \geq R_{2}$ AND $\Delta R_{1}=-\Delta R_{2}$.

\begin{tabular}{|c|c|c|c|c|c|c|c|}
\hline $\boldsymbol{R}_{\mathbf{1}}$ & $\boldsymbol{R}_{\mathbf{2}}$ & $\boldsymbol{R}_{\boldsymbol{A B m}}$ & $\boldsymbol{R}_{\boldsymbol{A B d}}$ & $\boldsymbol{R}_{\boldsymbol{D C m}}$ & $\boldsymbol{R}_{\boldsymbol{D C d}}$ & $\left|\Delta \boldsymbol{R}_{\boldsymbol{A B}}\right|$ & $\left|\Delta \boldsymbol{R}_{\boldsymbol{D C}}\right|$ \\
\hline $\boldsymbol{\Omega}$ & $\Omega$ & $\Omega$ & $\Omega$ & $\Omega$ & $\Omega$ & $\Omega$ & $\Omega$ \\
\hline 100.00 & 100.00 & 0.001 & 0.000 & -0.010 & 0.000 & 0.001 & 0.010 \\
\hline 100.20 & 99.80 & 0.393 & 0.400 & -0.023 & 0.000 & 0.007 & 0.023 \\
\hline 100.40 & 99.60 & 0.792 & 0.800 & -0.042 & 0.000 & 0.008 & 0.042 \\
\hline 100.60 & 99.40 & 1.193 & 1.200 & -0.058 & 0.000 & 0.007 & 0.058 \\
\hline 100.80 & 99.20 & 1.597 & 1.600 & -0.072 & 0.000 & 0.003 & 0.072 \\
\hline 101.00 & 99.00 & 2.003 & 2.000 & -0.085 & 0.000 & 0.003 & 0.085 \\
\hline
\end{tabular}

TABLE IV. RESULTS WHEN $R_{1}=R_{2}$ AND $\Delta R_{1}=\Delta R_{2} \geq 0$.

\begin{tabular}{|c|c|c|c|c|c|c|c|}
\hline $\boldsymbol{R}_{\mathbf{1}}$ & $\boldsymbol{R}_{\mathbf{2}}$ & $\boldsymbol{R}_{\boldsymbol{A B \boldsymbol { m }}}$ & $\boldsymbol{R}_{\boldsymbol{A B d}}$ & $\boldsymbol{R}_{\boldsymbol{D C} \boldsymbol{m}}$ & $\boldsymbol{R}_{\boldsymbol{D C d}}$ & $\mid \boldsymbol{\Delta \boldsymbol { R } _ { \boldsymbol { A B } } |}$ & $\mid \boldsymbol{\Delta \boldsymbol { R } _ { \boldsymbol { D C } } |}$ \\
\hline $\boldsymbol{\Omega}$ & $\boldsymbol{\Omega}$ & $\boldsymbol{\Omega}$ & $\boldsymbol{\Omega}$ & $\boldsymbol{\Omega}$ & $\boldsymbol{\Omega}$ & $\boldsymbol{\Omega}$ & $\boldsymbol{\Omega}$ \\
\hline 100.00 & 100.00 & 0.000 & 0.000 & 0.000 & 0.000 & 0.000 & 0.000 \\
\hline 100.20 & 100.20 & 0.002 & 0.000 & 0.401 & 0.400 & 0.002 & 0.001 \\
\hline 100.40 & 100.40 & 0.006 & 0.000 & 0.802 & 0.800 & 0.006 & 0.002 \\
\hline 100.60 & 100.60 & 0.007 & 0.000 & 1.202 & 1.200 & 0.007 & 0.002 \\
\hline 100.80 & 100.80 & 0.008 & 0.000 & 1.603 & 1.600 & 0.008 & 0.003 \\
\hline 101.00 & 101.00 & 0.010 & 0.000 & 2.002 & 2.000 & 0.010 & 0.002 \\
\hline
\end{tabular}

The system is dedicated to work with a pair of sensors whose changes in resistance have the same or opposite signs. As it can be seen in Table I-Table IV, for changes $\Delta R_{1}, \Delta R_{2}$ in range $\pm 1 \Omega$, slightly higher values of nonlinearity error $\left|\Delta R_{D C}\right|$ are obtained for sums than for differences (Table I-Table III), with exception of the case when both changes in resistance are of the positive signs (Table IV).

\section{CONCLUSIONS}

Comparing this $\mathrm{R} / \mathrm{U}$ transducer with well-known versions, the main conclusion is that switching current supply results in a more complicated measuring circuit and signals processing. On the other hand, the pulsed powering reduces thermoelectric voltages occurring on connections between the sensors, the amplifier and the ADC converter and low frequency noise by the use of differential outputs in the transducer.

The model presented in Fig. 2 contains some redundant elements on the PCB which should be eliminated in the final version of the transducer. Their presence is caused by testing other circuit configurations by using the same PCB.

On the basis of the measurements which were conducted with the use of an IR camera, we concluded that the supply part of the device (Fig. 8, right side) should be separated from the measuring part. The DC/DC converter dissipates heat to the PCB board. A direction of heat transfer is from the left side to the right side of the PCB (Fig. 2). The temperature of the PCB rises up about 15 minutes after turning the power on.

The results were obtained when the device had a constant temperature. The system was calibrated to be used with a pair of resistance decade boxes. In the case when $R_{1} \geq R_{2}$ and $\Delta R_{1}=-\Delta R_{2}$ the error values (Table III) are acceptable for $\mathrm{AB}$ (difference) channel (max. error is $0.8 \%$ ) and worse for DC (sum) channel ( $8.5 \%$ ). In the case when $R_{1}=R_{2}$ and $\Delta R_{1}=\Delta R_{2}>0$ (Table IV) these results are acceptable $(\leq 1 \%)$ for both channels. This second case can be considered to apply with real sensors. This transducer can be used for measuring very small differences in temperatures measured by two RTD sensors, for example in biomedical [11], industrial [12] systems or with differential sensor [13]. However, in these cases a thermal drift of the device need to be eliminated. The metrological parameters of the transducer are shown in Table $\mathrm{V}$.

TABLE V. METROLOGICAL PARAMETERS OF R/U TRANSDUCER.
\begin{tabular}{|c|c|}
\hline Bandwidth & $1 \ldots 100 \mathrm{~Hz}$ \\
\hline $\boldsymbol{R}_{\boldsymbol{A} B}, \boldsymbol{R}_{\boldsymbol{D} C}$ measuring range & $\pm 1 \Omega$ \\
\hline Max. nonlinearity error & $0.085 \Omega$ \\
\hline Sensitivity & $1.5 \mathrm{~V} / \Omega$ \\
\hline Signal noise & $2 \mathrm{mV}$ peak-to-peak \\
\hline Power supply & $15 \mathrm{VDC}, 420 \mathrm{~mA}$ \\
\hline
\end{tabular}

\section{REFERENCES}

[1] F. Reverter, O. Casas, "Interfacing differential resistive sensors to microcontrollers: a direct approach", IEEE Trans. Instrumentation and Measurement, vol. 58, no. 10, pp. 3405-3410, 2009. [Online]. Available: http://dx.doi.org/10.1109/TIM.2009.2017651

[2] B. Maundy, S. J. G. Gift, "Strain gauge amplifier circuits", IEEE Trans. Instrumentation and Measurement, vol. 62, no. 4, pp. 693-700, 2013. [Online]. Available: http://dx.doi.org/10.1109/TIM.2013. 2246904

[3] M. M. S. Anands, Electronic Instruments and Instrumentation Technology. Prentice-Hall of India, New Delhi, 2006.

[4] G. S. Zhao, H. F. Pei, H. C. Liang, "Measurement of additional strains in shaft lining using differential resistance sensing technology", Int. Journal of Distributed Sensor Networks, 2013. [Online]. Available: http://dx.doi.org/10.1155/2013/153834

[5] Y. Kervran, O. De Sagazan, S. Crand, N. Coulon, T. MohammedBrahim, O. Brel, "Microcrystalline silicon: Strain gauge and sensor arrays on flexible substrate for the measurement of high deformations", Sensors and Actuators A: Physical, vol. 236, pp. 273280, 2015. [Online]. Available: http://dx.doi.org/10.1016/j.sna. 2015.08.001

[6] D. Milkovic, G. Simic, Z. Jakovljevic, J. Tanaskovic, V. Lucanin, "Wayside system for wheel-rail contact forces measurements", Measurement, vol. 46, no. 9, pp. 3308-3318, 2013. [Online]. Available: http://dx.doi.org/10.1016/j.measurement.2013.06.017

[7] A. Idzkowski, P. Swietochowski, W. Walendziuk, Z. L. Warsza, "Unconventional double $\mathrm{R} / \mathrm{U}$ converter for measurement of two quantities by a single differential sensor", Advances in Intelligent Systems and Computing, vol. 352, pp. 83-90, 2015. [Online]. Available: http://dx.doi.org/10.1007/978-3-319-15835-8_10

[8] Z. L. Warsza, "Bridges supplied by two current sources-new tool for impedance measurements and signal conditioning", in Proc. IMEKOTC-7 Symposium, Cracow, Poland, 2002, pp. 231-236.

[9] A. Idzkowski, W. Walendziuk, Z. L. Warsza, "Unconventional double-current circuit for deflection and temperature simultaneous measurement", Elektronika ir Elektrotechnika, vol. 21, no. 1, pp. 2327, 2015. [Online]. Available: http://dx.doi.org/10.5755/j01.eee. 21.1.6834

[10] Noise reduction and isolation, technical articles of Measurement Computing. [Online]. Available: http://www.mecdaq.com/technicalarticles.aspx

[11] T. Otahalova, Z. Slanina, D. Vala, "Embedded sensors system for real time biomedical data acquisition and analysis", 11th IFAC/IEEE Int. Conf. Programmable Devices and Embedded Systems (PDeS), 2012. [Online]. Available: https://doi.org/10.3182/20120523-3-CZ-3015. 00050

[12] Z. L. Warsza, A. Idzkowski, "About accuracy of the simultaneous measurement of temperature difference and average using transducers with Pt sensors - O dokładności układów z czujnikami Pt do równoczesnych pomiarów różnicy temperatur i ich wartości średniej”, Przemysl Chemiczny, vol. 96, no. 2, pp. 278-283, 2017. [Online]. Available: http://dx.doi.org/10.15199/62.2017.2.20. (in Polish).

[13] S. Ali, A. Hassan, J. Bae, C. H. Lee, J. Kim, "All-printed differential temperature sensor for the compensation of bending effects", Langmuir, vol. 32, no. 44, pp. 11432-11439, 2016. [Online]. Available: http://pubs.acs.org/doi/abs/10.1021/acs.langmuir.6b02885 